\title{
Efeitos da Imprevisibilidade Familiar e das Diferenças em Função do Sexo Sobre a Propensão ao Risco, Exposição à Violência e o Desconto do Futuro de Jovens Universitários: Uma Abordagem Evolucionista
}

\author{
Alice Andrade Silva* \\ Rosana Suemi Tokumaru \\ Universidade Federal do Espírito Santo, Vitória, ES, Brasil \\ Anna Beatriz Carnielli Howat-Rodrigues \\ Universidade de São Paulo, São Paulo, SP, Brasil
}

\begin{abstract}
RESUMO
Partindo da abordagem evolucionista objetivou-se identificar efeitos da imprevisibilidade familiar na infância e das diferenças em função do sexo sobre propensão ao risco, expectativa de futuro e percepção de exposição à violência. Participaram 233 jovens (117 homens, M=18,96 anos). O grupo com maior imprevisibilidade na infância apresentou maior risco de competição e expectativa de vida. Homens apresentaram maiores taxas de propensão ao risco e exposição direta à violência; mulheres maiores taxas de exposição indireta à violência. Concluiu-se que imprevisibilidade familiar e diferenças em função do sexo não apresentaram efeitos lineares sobre as variáveis estudadas, como previsto a partir da abordagem evolucionista. Aponta-se a necessidade de integrar teorias biológicas, psicológicas e sociais e de desenvolver instrumentos adaptados para a população brasileira.
\end{abstract}

Palavras-chave: risco; sexo; imprevisibilidade familiar.

\section{ABSTRACT \\ Effects of Family Unpredictability and Sexual Differences on Risk-taking, Violence Exposure and Future Discounting of College Students: An Evolutionary Perspective}

From an evolutionary perspective, this study aimed to identify effects of sex differences and childhood family unpredictability on risk-taking, future discounting and perceptions of exposure to violence among young people. Participants were 233 people (117 men, $M=18.96$ years). The group with higher childhood family unpredictability presented higher risk of competition and life expectancy. Men presented higher rates of risk-taking and direct exposure to violence; women presented higher rates of indirect exposure to violence. We concluded that family unpredictability and sex differences did not show linear effects on the variables studied, as predicted from the evolutionary approach. The need to integrate biological, psychological and social theories and to develop appropriate instruments for the Brazilian population is emphasized.

Keywords: risk; sex characteristics; family unpredictability.

\section{INTRODUÇÃO}

Comportamentos de risco ocorrem em diferentes contextos e em diferentes momentos da vida das pessoas (Wang, Kruger, \& Wilke, 2009). São considerados comportamentos de risco, por exemplo, as práticas sexuais sem preservativos e com muitos parceiros (Falcão Júnior, Lopes, Freitas, Rabelo, Pinheiro, \& Ximenes, 2007), a ingestão excessiva de álcool e o uso de outras drogas, se envolver em brigas com amigos ou em agressões físicas ou verbais (Pillon, O'Brien \& Piedra, 2005). A tendência a tomar decisões que resultem em potenciais danos físicos, psicológicos ou de sobrevivência em busca de uma recompensa é denominada propensão ao risco (Byrnes, Miller, \& Schafer, 1999; Daly \& Wilson, 2001; Feijó \& Oliveira, 2001; Howat-Rodrigues, 2010; Koller, 1999; 
Ross \& Hill, 2002). A literatura aponta que a propensão ao risco pode ser influenciada pela expectativa de futuro do indivíduo, pelas diferenças em função do sexo e por aspectos socioeconômicos e culturais. (Daly \& Wilson, 2005; Hill, Jenkins \& Farmer, 2008; Ross \& Hill, 2002; Ross, Hill \& Low, 1997; Falcão Júnior et al, 2007).

Magar, Phillips e Hosie (2008) consideram que a preferência por riscos resulta do mau funcionamento cognitivo de avaliação e baixa regulação emocional. Entretanto, à luz da psicologia evolucionista, arriscar-se para adiantar ganhos ou conseguir recursos pode ser uma forma de evitar o risco de perdê-los e pode ser uma resposta funcional e adaptativa (Wilson \& Daly, 1997, Daly \& Wilson, 2005; Ross \& Hill, 2002). Arriscar-se para obter recursos pode ter sido particularmente estratégico considerando ambientes altamente imprevisíveis. Em tais ambientes o comportamento de risco aumentaria as chances de obtenção de recursos no momento em que estão disponíveis (Daly \& Wilson, 2001; Wang et al., 2009). O envolvimento de jovens em crimes e a gravidez na adolescência foram abordados sob este aspecto (Daly \& Wilson, 2001, Wilson \& Daly, 1997; 2006; Wilson, Daly, \& Pound, 2002). Quanto maior a imprevisibilidade sobre a disponibilidade de recursos maior seriam as preferências por ganhos imediatos, mesmo que menores e envolvendo maiores riscos. Essa preferência por ganhos menores, porém mais imediatos, tem sido denominada de desconto do futuro (Wilson \& Daly, 2004).

A propensão ao risco pode sofrer influência de características pessoais, como idade e sexo (Baker \& Maner, 2008; Daly \& Wilson, 2005; Hill et al, 2008; Howat-Rodrigues, 2010; Ross et al, 1997) e ambientais, como a imprevisibilidade atual, a imprevisibilidade familiar na infância, a exposição à violência por meio da mídia, os valores ensinados pelos pais e grupos sociais e a vulnerabilidade social (Davis \& Werre, 2008; Ross \& Hill, 2002; Wilson \& Daly, 1997; Feijó \& Oliveira, 2001; Aldrighi, 2004; Brandão \& Heilborn, 2006; Vermelho, Barbosa, \& Nogueira, 1999). Além disso, tanto a propensão ao risco quanto a expectativa de futuro entre jovens podem estar relacionados com a percepção de um ambiente violento e com a execução de comportamentos agressivos e violentos (Cara \& Gauto, 2007; Davis \& Werre, 2008).

Agressões físicas (assassinato, espancamento, uso de arma de fogo, por exemplo) ou verbais (gritar, usar palavras hostis, briga entre amigos e familiares, por exemplo) (Pillon et al., 2005) são classificadas nesse estudo como violência direta - se perpetradas ou sofridas pelo indivíduo no âmbito familiar ou social mais amplo; e violência indireta - se testemunhada ou percebida através de pessoas conhecidas ou da mídia. Entre jovens universitários as mulheres foram as maiores vítimas de coerção sexual e psicológica no relacionamento, mas, na agressão física ao companheiro homens e mulheres não se diferenciaram (Aldrighi, 2004). Os jovens universitários do sexo masculino foram os que mais se envolveram com brigas entre amigos e com policiais, seja sofrendo agressões ou perpetrando, além de se envolver em outros comportamentos de risco como dirigir embriagado, fazer sexo sem camisinha com várias parcerias e ingerir bebidas alcoólicas exageradamente (Pillon et al., 2005).

Embora se considere uma vasta literatura que aponta a influência de uma variedade de fatores sobre o comportamento de risco ressaltamos que esse estudo desenvolve a questão sob a perspectiva da psicologia evolucionista e nesse sentido ocupa-se da influência das diferenças em função do sexo e da imprevisibilidade familiar. Parte-se do pressuposto de que o comportamento de risco pode ser uma resposta adaptativa ao contexto, como parte de uma estratégia reprodutiva, e estimulada pela percepção de imprevisibilidade na tentativa de aumentar a probabilidade de adiantamento dos ganhos de uma ação.

\section{Diferenças em função do sexo}

De acordo com a teoria do Investimento Parental, diferenças em função do sexo existem como estratégias para maximizar o sucesso reprodutivo (Trivers, 1972). A estratégia de reprodução masculina envolveria o maior esforço de busca de parceiras - os homens tenderiam a disputar recursos para ter acesso ao maior número possível de parcerias, pois, desta forma, maximizariam seu potencial reprodutivo. A estratégia de reprodução feminina envolveria o maior esforço parental devido ao custoso investimento energético que faz mediante o óvulo e a gestação (Krebs \& Davies, 1996; Ross et al., 1997). Assim, considera-se que o esforço para encontrar o maior número de parceiras sexuais envolve mais risco enquanto o esforço parental, mais cautela e, portanto, espera-se que os homens apresentem maiores taxas de propensão ao risco do que as mulheres.

Muitas pesquisas confirmam a hipótese de que homens são mais propensos ao risco, mais expostos a situações de violência e preferem adiantar ganhos a ter 
que esperar certo tempo para recebê-los. Segundo Wang et al. (2009), a estratégia reprodutiva masculina resulta em alta taxa de mortalidade entre jovens do sexo masculino devido à propensão ao risco de competição intergrupal e intragrupal por status, alocação de recursos e acesso a parceiras sexuais. Fetchenhauer e Rohde (2002) encontraram que os homens fazem mais escolhas voltadas para o presente e escolhas que envolvem risco do que mulheres e relacionaram esses dados com o fato de que ao longo da história evolutiva os homens se envolveram num nível mais elevado de concorrência intra-sexual. Gava e Vieira (2008) em estudo sobre o comportamento do investidor e sua tolerância ao risco concluíram que homens eram mais tolerantes ao risco do que mulheres, o que contribuiu para que se arriscassem mais. Além disso, a maior parte dos casos de homicídios, suicídios, do uso de drogas e de acidentes de trânsito é vivenciada por jovens/adolescentes do sexo masculino (Baus, Kupek, \& Pires, 2002; Lyra, Goldberg \& Iyda, 1996; Souza, Assis \& Silva, 1997; Waiselfisz, 2012, 2013; Yunes \& Primo, 1983).

Considerando os aspectos socioeconômicos e culturais, Souza (2005) enfatiza que essas associações entre o masculino e a violência são constituídas em práticas machistas e de risco que colocam os jovens do sexo masculino entre as maiores vítimas de violência e entre as pessoas que menos assumem seus medos e incertezas. Ao mesmo tempo, o feminino é visto como o sexo frágil, mais exposto ao perigo e menos apto a se defender. Estatisticamente, as mulheres são mais frequentemente vítimas da violência sexual, física e psicológica (Aldrighi, 2004; Kronbauer \& Meneghel, 2005). Assim, fatores contextuais e culturais parecem contribuir para a ocorrência de diferenças em função do sexo no comportamento de risco, sendo que o gênero masculino se apresenta mais associado ao comportamento de risco que o gênero feminino.

\section{Imprevisibilidade familiar na infância}

A partir das teorias do apego inseguro e do desamparo aprendido, Ross e Hill (2002) criaram o conceito de imprevisibilidade familiar na infância, determinada pela inconsistência no fornecimento de carinho, regras, disciplina e recursos financeiros para a criança (Ross et al., 1997). Pressupõe-se que o padrão de comportamento familiar inconsistente subsidia um modelo mental de imprevisibilidade levando os indi- víduos a exibirem aumento na propensão ao risco como forma de adiantar recursos.

Alguns resultados dão suporte a esta hipótese. Wilson \& Daly (1997) encontraram relação negativa entre a taxa de homicídios de diferentes bairros de Chicago e a expectativa de vida de seus habitantes. A diminuição da expectativa de vida, por sua vez, relacionou-se positivamente com a antecipação do inicio da vida reprodutiva. Os autores pressupõem que a taxa de homicídios reflita a imprevisibilidade do ambiente e que, quanto maior a imprevisibilidade, maior o desconto do futuro, representado pela antecipação do ciclo reprodutivo. Surbey (1990) demonstrou que meninas que crescem num ambiente imprevisível, indicado pela falta de um dos pais e de altos índices de mortalidade juvenil, adiantaram a menarca e iniciaram sua vida sexual precocemente. Wilson e Daly (2006) demonstraram que meninas e meninos que estiveram mais expostos à violência e à instabilidade na trajetória escolar se tornaram infratores com mais frequência e tornaram-se pais mais cedo do que aqueles que estiveram expostos a menos violência e instabilidade na vida escolar. Falcão Júnior et al. (2007) indicaram que jovens universitários do curso de enfermagem também assumiram práticas sexuais sem uso de preservativo colocando-se em risco de contaminação por DST/AIDS. Os autores destacaram que ter informação sobre DST/AIDS não preveniu o comportamento de risco e apontaram como fatores que contribuem para o risco o tipo de vínculo com o parceiro e a idade dos participantes. A imprevisibilidade ainda tem sido associada à agressividade entre homens (Ross et al., 1997) e ao uso de substâncias psicotrópicas (Daly \& Wilson, 2001; Ross \& Hill, 2002; Sanchez, Oliveira, \& Nappo, 2005).

Diante do exposto, o objetivo deste estudo foi identificar diferenças em função do sexo e de imprevisibilidade familiar na infância sobre a propensão ao risco, a expectativa de futuro e a percepção de exposição à violência entre jovens universitários. De acordo com a literatura esta faixa etária apresenta maior propensão ao risco e é mais orientada em direção a recompensas imediatas se comparada com pessoas consideradas adultas (Daly \& Wilson, 2005; Wilson et al., 2002; Steinberg, 2004). Além disso, a população universitária, geralmente, vivencia profundo processo de mudança, com o afastamento da família e a inserção em grupos de amigos onde há maior pressão para usar bebidas alcoólicas, fazer sexo sem proteção e dirigir embriagado (Pillon et al., 2005). 
Trabalhou-se com a hipótese de que ambientes percebidos como mais imprevisíveis estariam diretamente relacionados a altas taxas de propensão ao risco, de percepção de exposição à violência e de baixa expectativa de futuro. Em relação às diferenças em função do sexo, espera-se que os homens sejam mais propensos ao risco e percebam maior exposição à violência. Em relação à expectativa de futuro, esperase que homens, mais do que mulheres, teriam uma perspectiva temporal orientada para o presente.

\section{METODOLOGIA}

\section{Participantes}

Participaram da pesquisa 233 jovens universitários de uma universidade do Espírito Santo, 50,2\% do sexo masculino ( $n=117)$ e $49,8 \%$ do sexo feminino $(n=116)$, graduandos dos cursos de administração, engenharia, língua portuguesa, psicologia e serviço social, com uma idade média de 18,96 anos ( $\mathrm{dp}=1,62)$. O trabalho foi realizado com amostra de conveniência. De acordo com o critério de jovem, adotado pelo Instituto Brasileiro de Geografia e Estatística - IBGE (1999) - pessoas entre os 15 e 24 anos, decidiu-se pela delimitação da amostra a esta faixa etária. O poder de compra (CCEB - ABEP, 2008) indicou que $43,1 \%$ dos participantes pertenciam à classe $\mathrm{B}, 30,2 \%$ à classe $\mathrm{C}, 25,3 \%$ à classe $\mathrm{A}$ e $1,4 \%$ à classe $\mathrm{D}$.

\section{Instrumentos}

O questionário foi composto por:

(a) questões sociodemográficas para caracterização dos participantes (sexo, idade e escolaridade) e os escores do Critério de Classificação Econômica Brasil (CCEB) que fornece informações sobre o poder de compra dos participantes (ABEP, 2008).

(b) medida de expectativa de futuro, composta pelo escore individual de desconto do futuro e duas questões sobre expectativa de futuro: expectativa de vida (“Até que idade você imagina que vai viver?") e percepção de futuro ("Daqui a quanto tempo é futuro para você?"). O escore de desconto do futuro foi obtido a partir das respostas individuais a nove questões sobre preferência por receber valores menores em um prazo mais curto ou maiores em um prazo mais longo (por exemplo, "Você prefere receber $\mathrm{R} \$ 34,00$ amanhã ou $\mathrm{R} \$ 35,00$ em 186 dias?”). O procedimento de cálculo do desconto foi baseado numa equação de função hiperbólica, denominado valor k (Kirby \& Santiesteban, 2003, Wilson \& Daly, 2004). Esse instrumento não apresenta normalização para respondentes brasileiros, mas tem sido utilizado em vários estudos mostrando adequação de construto (Ferreira, 2009; Ornellas, 2010; Howat-Rodrigues, 2010).

(c) propensão ao risco: Escala de Propensão ao Risco Específico - Domínios Específicos (EPRE DE). Validada para a amostra brasileira por HowatRodrigues, De Andrade e Tokumaru (2013), o instrumento é constituído de 14 itens autoavaliados em escala Likert de 5 pontos ( $1=$ nunca faria; $5=$ sempre faria) baseada em dois fatores evolutivos: coesão (Alpha de Cronbach $=0,82$ ), incluindo itens que expressam comportamentos individuais, de acordo com normas explícitas ou implícitas do grupo parental ou não, os quais favorecem a permanência, status e estabilidade do indivíduo no mesmo ("Usar drogas ilegais); e competição (Alpha de Cronbach =0,60), incluindo itens que refletem a interação do indivíduo com seu grupo ou grupos diferentes na disputa por recursos como alimento, poder/status, entre outros ("Cair na porrada com amigo, familiar ou conhecido).

(d) percepção de exposição à violência: A Escala de Percepção de Exposição à Violência (EPEV) foi validada para amostra brasileira por Lima, HowatRodrigues e Tokumaru (no prelo). É composta por 9 itens divididos em dois fatores: (1) exposição indireta à violência ("Ver ou saber de brigas, porradas") e (2) exposição direta à violência ("Atirar em alguém"). Os itens da EPEV foram autoavaliados em escala Likert de 5 pontos ( $1=$ nunca, $5=$ sempre). A EPEV foi construída usando como critério classe social e sexo, e nesse sentido apresenta uma característica interessante e inovadora: permite o estudo de temas mais abrangentes por avaliar a exposição à violência na população em geral (Lima et al., no prelo).

e) Imprevisibilidade no contexto infantil: Escala de Imprevisibilidade Familiar na Infância (EIFI). Validado para amostra brasileira por Howat-Rodrigues, De Andrade e Tokumaru (2012) o instrumento é constituído de 27 itens autoavaliados em escala Likert de 5 pontos ( $1=$ discordo totalmente; $5=$ concordo totalmente) baseada em 4 fatores: cuidado/apoio (Alpha de Cronbach $=0,88$; "Eu tinha certeza que minha família cuidaria de mim"), recursos financeiros (Alpha de Cronbach $=0,85$; "Houve momentos em minha infância em que tínhamos pouco dinheiro para viver"), 
alimentação (Alpha de Cronbach =0,80; "Na minha casa o horário das refeições era diferente a cada dia") e disciplina (Alpha de Cronbach $=0,78$; "Na minha casa a disciplina que meus familiares me davam era inconsistente").

\section{Coleta de dados e aspectos éticos do estudo}

Seguindo as normas do Conselho Nacional de Saúde (CNS 196/96), a proposta de pesquisa foi aprovada pelo Comitê de Ética em Pesquisa com Seres Humanos (CEPSH) da Universidade Federal do Espírito Santo (Processo $n^{\circ}$ 089/09). A coleta de dados foi realizada em sessões coletivas em sala de aula, após autorização dos professores. Os universitários que consentiram em participar assinaram o Termo de Consentimento Livre e Esclarecido.

\section{Procedimento de análise de dados}

Os dados foram tabulados e analisados estatisticamente no SPSS versão 16. Propensão ao risco, exposição à violência e expectativa de futuro foram tratados como construtos independentes, desta forma, avaliouse a correlação entre os indicadores de cada construto entre si utilizando a correlação de Pearson. Os indicadores de imprevisibilidade familiar na infância foram submetidos a uma análise de Cluster, com o objetivo de identificar grupos homogêneos com base em suas características (Hair, Anderson, Thatam, \& Black, 2006). Para avaliar o efeito da imprevisibilidade e do sexo dos participantes sobre a propensão ao risco, a exposição à violência e a expectativa de futuro foram utilizadas análises de GLM (General Linear Models MANOVA e ANOVA - Dancey \& Reidy, 2006).

\section{RESULTADOS}

Indicadores de propensão ao risco (competição e coesão; $r=0,370 ; p<0,001$ ) estiveram significativamente correlacionados entre si, bem como os indicadores de exposição à violência (direta e indireta; $r=0,323 ; p<0,001)$. Os indicadores de expectativas de futuro (desconto do futuro, expectativa de vida e tempo futuro) não apresentaram correlação significativa entre si (desconto do futuro e expectativa de vida $r=0,028$; desconto do futuro e quando é futuro $r=0,041$; expectativa de vida e quando é futuro $-r=-$ 0,031). Foram, portanto, utilizados como variáveis independentes. Aplicou-se Análise de Cluster com o propósito de identificar grupos homogêneos em relação à imprevisibilidade familiar na infância. De acordo com a Tabela 1, três grupos foram formados.

Tabela 1

Descrição dos Clusters

\begin{tabular}{|c|c|c|c|c|}
\hline Grupo/nome & Variável & Média (dp) & Descrição & No de casos \\
\hline 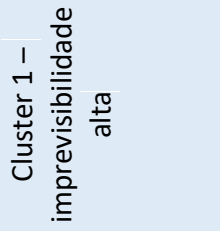 & $\begin{array}{c}\text { Disciplina } \\
\text { Alimentação } \\
\text { Recursos Financeiros } \\
\text { Cuidado }\end{array}$ & $\begin{array}{l}1,52(0,54) \\
3,29(0,60) \\
2,47(0,91) \\
1,52(0,40)\end{array}$ & $\begin{array}{l}\text { O primeiro conglomerado relaciona-se a casos } \\
\text { com maior imprevisibilidade de alimentação, } \\
\text { recursos financeiros e cuidado e imprevisibilidade } \\
\text { média em disciplina. }\end{array}$ & 48 \\
\hline 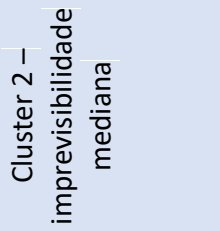 & $\begin{array}{c}\text { Disciplina } \\
\text { Alimentação } \\
\text { Recursos Financeiros } \\
\text { Cuidado }\end{array}$ & $\begin{array}{l}3,26(0,63) \\
2,72(0,85) \\
2,31(0,81) \\
1,71(0,65)\end{array}$ & $\begin{array}{l}\text { O segundo conglomerado agrupou casos com } \\
\text { maior imprevisibilidade em disciplina, menor } \\
\text { imprevisibilidade em recursos financeiros e } \\
\text { imprevisibilidade mediana em alimentação e } \\
\text { cuidado. }\end{array}$ & 51 \\
\hline 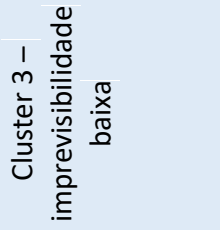 & $\begin{array}{c}\text { Disciplina } \\
\text { Alimentação } \\
\text { Recursos Financeiros } \\
\text { Cuidado }\end{array}$ & $\begin{array}{l}1,22(0,41) \\
1,88(0,50) \\
1,65(0,59) \\
1,27(0,35)\end{array}$ & $\begin{array}{l}\text { O terceiro conglomerado agrupou casos com } \\
\text { menor imprevisibilidade em disciplina, } \\
\text { alimentação e cuidado e imprevisibilidade média } \\
\text { em recursos financeiros. }\end{array}$ & 123 \\
\hline
\end{tabular}

Diferenças entre grupos: imprevisibilidade familiar na infância e diferenças em função do sexo.
Foram analisadas as diferenças entre os grupos de imprevisibilidade familiar na infância (alta, mediana e 
baixa) e diferenças em função do sexo (homem versus mulher) em relação à propensão ao risco, exposição à violência e expectativa de futuro. Houve diferença entre os grupos de imprevisibilidade (Tabela 2) apenas quanto à propensão ao risco e a expectativa de vida. As análises subsequentes (post hoc) de comparação (pairwise comparisons, ajustadas pelo método de Bonferroni) mostraram que o grupo com maior im- previsibilidade familiar na infância apresentou o maior risco de competição enquanto o grupo com menor imprevisibilidade familiar na infância apresentou o menor risco de competição. Quanto à expectativa de vida, o grupo com imprevisibilidade alta teve maior expectativa de vida do que o de imprevisibilidade média, sendo essa diferença de 9,87 anos.

\section{Tabela 2}

Comparações dos indicadores de propensão ao risco (MANOVA), exposição à violência (MANOVA) e expectativa de futuro (ANOVA) entre os grupos de imprevisibilidade familiar na infância

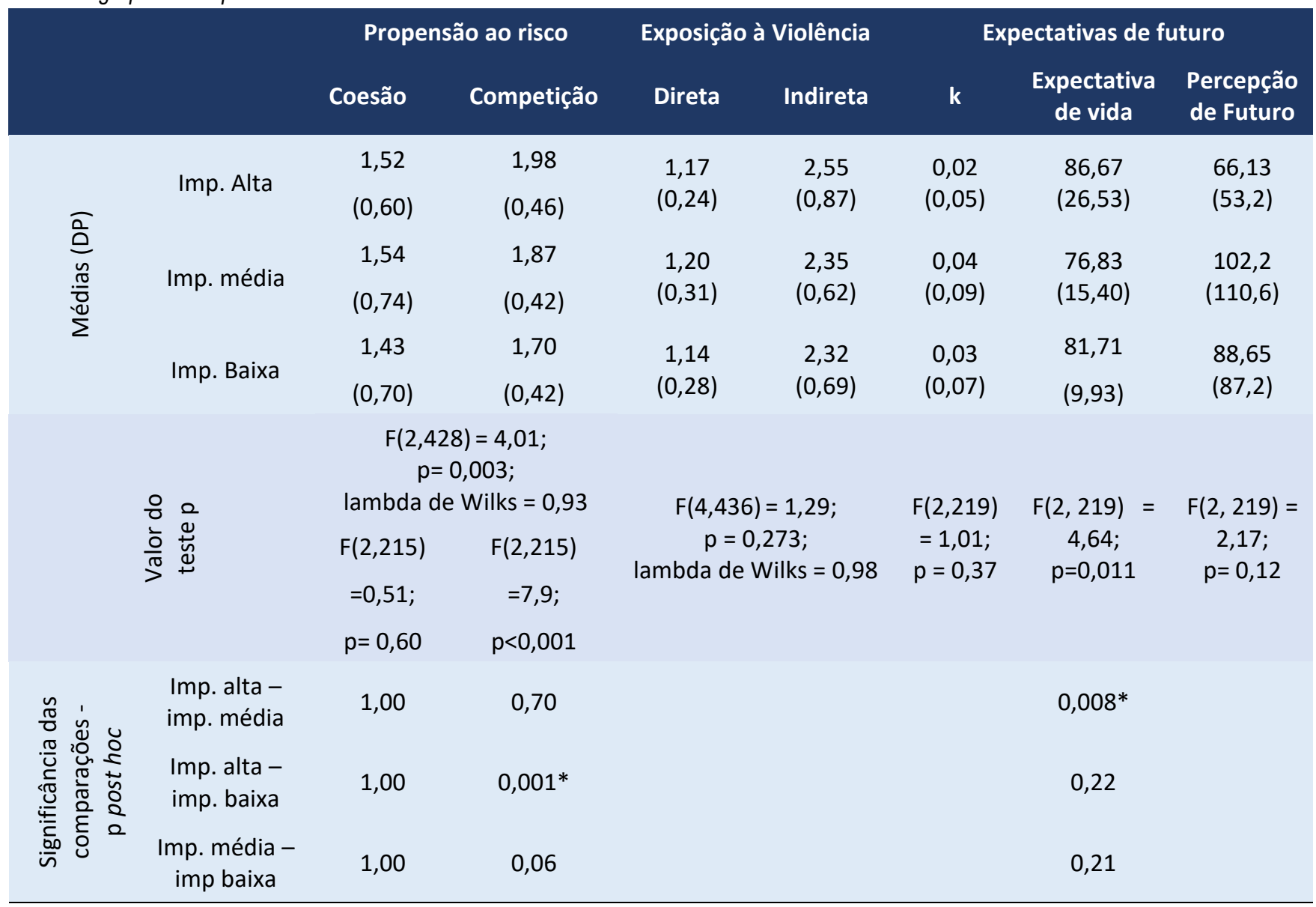

Nota. * Significância $<0.05, * *$ Significância $<0.001$

Houve diferença estatisticamente significativa entre os sexos em indicadores de propensão ao risco e exposição à violência (Tabela 3). As análises subsequentes (post hoc) de comparação (pairwise compari- sons, ajustadas pelo método de Bonferroni) apontaram que homens apresentaram maiores taxas de propensão ao risco e mulheres apresentaram maiores taxas de exposição indireta à violência. 
Tabela 3

Comparações dos indicadores de propensão ao risco (MANOVA), exposição à violência (MANOVA) e expectativas de futuro considerando o sexo

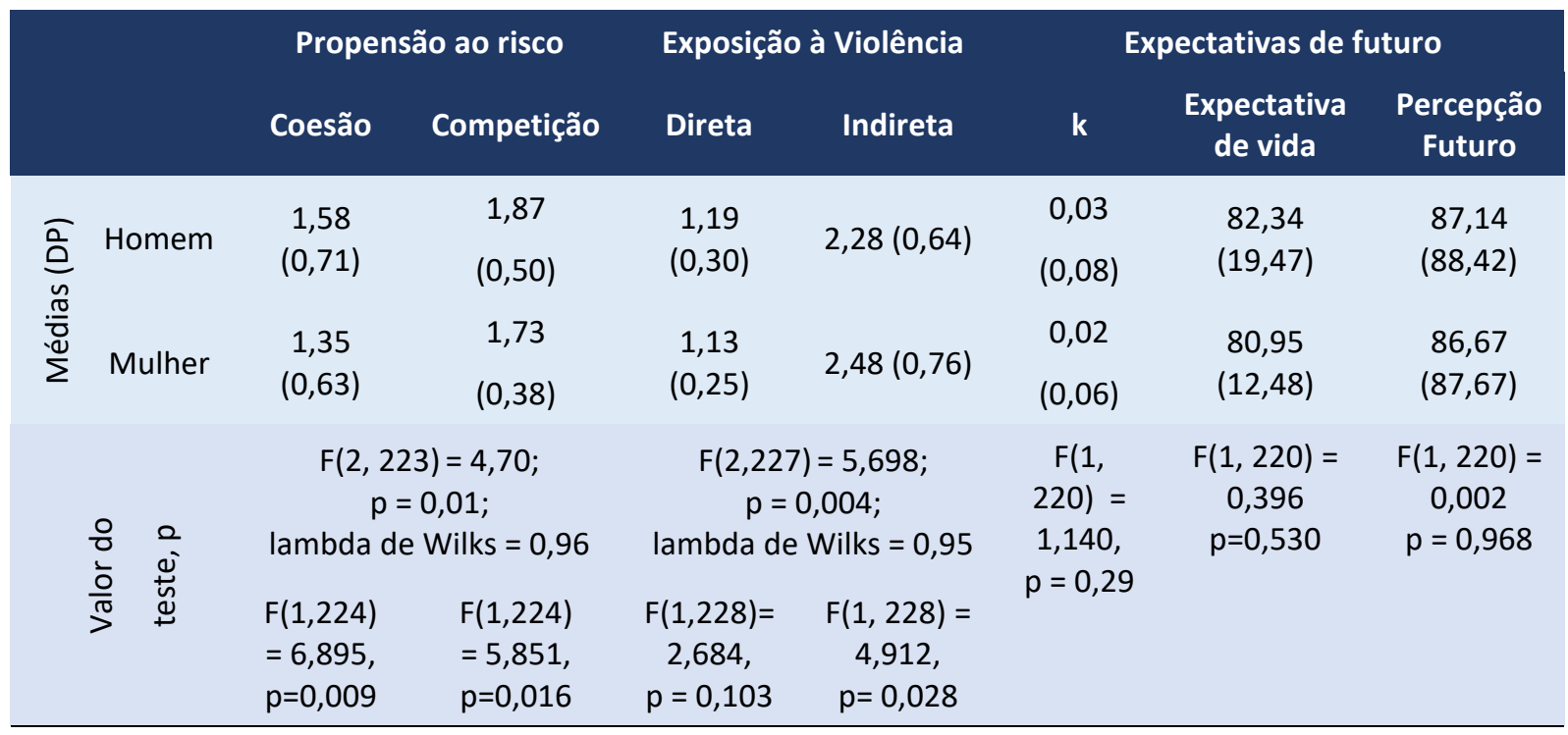

\section{DISCUSSÃO}

Trabalhamos com a hipótese inicial de que a percepção de maior imprevisibilidade na infância estaria relacionada a altas taxas de propensão ao risco e exposição à violência e expectativas de futuro baixas. Em relação a diferenças em função do sexo, homens seriam mais propensos ao risco, teriam uma perspectiva temporal mais orientada para o presente e igualmente, seriam mais envolvidos em exposição à violência. Os resultados apresentados nesse trabalho corroboraram parcialmente essas hipóteses: homens tiveram maiores taxas de propensão ao risco, todavia, não houve diferença significativa entre homens e mulheres quanto às expectativas de futuro. E, embora os homens tenham apresentado maiores médias quanto à exposição direta à violência, também não houve diferenças significativas em função do sexo. Como resultado não previsto a partir da hipótese inicial, mulheres apresentaram maiores índices de exposição indireta à violência.

Índices de propensão ao risco de coesão e de competição foram maiores entre jovens do sexo masculino. De acordo com a literatura a estratégia reprodutiva masculina envolve maior nível de competição intrasexual apontada como motivo de violência entre jovens e incentivada pela concorrência por recursos, status ou parceria sexual (Aldrighi, 2004; Fetchenhauer \& Rohde, 2002; Wang et al., 2009; Wilson \&
Daly, 1997). Ainda, a estratégia sexual masculina também pode envolver a construção de alianças, o que leva os indivíduos a se engajarem em comportamentos típicos de algum grupo a fim de que, fazendo alianças e afirmando uma identidade grupal, tenham maiores chances de garantirem recompensas ou recursos (Daly \& Wilson, 2001; Feijó \& Oliveira, 2001).

Diferenças em função do sexo não estiveram significativamente relacionadas às taxas de expectativa de futuro, incluindo a taxa de desconto do futuro. Argumenta-se que as diferenças em função do sexo parecem ter sido selecionadas num ambiente ancestral, onde não existiram dilemas financeiros e que por isso não seria possível encontrar correlações usando esse instrumento (Ross et al., 1997). Ou que o método de escolhas monetárias usado como uma das medidas da expectativa de futuro tratou-se de uma medida cognitiva do risco e, por serem hipóteses imaginadas ao invés de reais, dificultou as correlações esperadas (Hill et al., 2008). Além disso, de acordo com HowatRodrigues (2010), estes resultados podem apontar para problemas de adaptação de instrumento para a população brasileira, visto que o instrumento ainda esta em fase de construção e discussão e não possui modelo já consolidado para respondentes brasileiros. Trabalhos que utilizaram este instrumento em populações brasileiras apresentaram diferentes resultados. Ferreira (2009) e Ornelas (2010) utilizaram o mesmo procedimento em uma amostra composta por universi- 
tários. Enquanto Ferreira (2009) encontrou diferença entre os sexos no desconto do futuro como esperado a partir da literatura, Ornelas (2010), assim como nesse estudo, não encontrou diferença entre homens e mulheres. Além da imprevisibilidade familiar na infância, levanta-se a possibilidade de outras variáveis contextuais influenciarem o desconto tornando homens e mulheres mais semelhantes, por exemplo, é possível que a homogeneidade da nossa amostra quanto à classe econômica (43,1\% são da Classe B) e os baixos níveis de imprevisibilidade (cerca de 55\%) tenha influenciado este resultado. Entretanto, estudos futuros que verifiquem a possibilidade de efeitos de interação entre variáveis contextuais e pessoais sobre o desconto do futuro poderão contribuir para compreender estes resultados.

Há controvérsias na literatura sobre a existência de diferenças em função do sexo na prática de violência e nessa amostra, homens e mulheres não diferiram quanto a esta variável no que diz respeito à exposição direta à violência. Embora muitos dados destaquem o sexo masculino como o maior perpetrador e o mais exposto à violência, outras pesquisas mostram índices de exposição direta à violência mútua. No geral, pesquisas apontam os homens como os que mais vitimizam e apresentam comportamentos de violência (Cardia, 1999; Daly \& Wilson, 2001; Waiselfisz, 2012, 2013). Segundo dados estatísticos, $95 \%$ do cárcere brasileiro é composto pelo sexo masculino (Rolim, 2009) e $91,4 \%$ dos homicídios registrados no ano de 2010 pertenciam ao sexo masculino (Waiselfisz, 2012). Nesse sentido "a vitimização no país é notadamente e fundamentalmente masculina" (Waiselfisz, 2013, p. 69), sendo que em 2013 os índices de prática de homicídio foram duplicados, juntamente com o aumento de $17,2 \%$ da vitimização feminina na década (Waiselfisz, 2013). Souza (2005, p.9) identifica que comportamentos violentos "são influenciados por determinantes socioeconômicos e culturais que de alguma forma potencializam a associação entre a masculinidade e a violência". De outra forma, Aldrighi (2004), trabalhando com amostra universitária, mostrou que $78,6 \%$ da prática de agressões físicas de maior severidade são práticas mútuas entre homens e mulheres que são/foram casal. Supomos que a ausência de diferença entre homens e mulheres na nossa amostra na exposição à violência direta também possa estar associada a homogeneidade da amostra quanto à classe econômica e aos baixos níveis de imprevisibili- dade, como sugerido para explicar a ausência de diferenças de desconto entre os sexos. A predominância destas características pode resultar em menor envolvimento em comportamentos de violência e na priorização da formação acadêmica e profissional, características apontadas por Saraiva \& Quixadá (2010) como parte do contexto de disciplina de universitários.

Embora não haja diferenças em função do sexo significativas quanto à exposição à violência, destacase o fato de que as maiores médias de exposição indireta à violência foram das mulheres. Esse resultado corrobora dados de pesquisas anteriores que mostram que mulheres se sentem mais expostas à violência pelo estereótipo cultural de fragilidade feminina e por sofrerem mais com a violência nos próprios relacionamentos (Aldrighi, 2004; Kronbauer \& Meneghel, 2005). De acordo com dados divulgados pelo Instituto Jones dos Santos Neves (2008), a Delegacia Especializada da Mulher no Espírito Santo [DEAM] (2008) registrou que o âmbito onde mais ocorre a violência contra mulher é dentro de casa - 70\%. Porém, os dados estatísticos apontam que são os homens as maiores vítimas de violência em âmbito geral. Os dados do Instituto Brasileiro de Geografia e Estatística (2010) mostram que o percentual de vitimização através de agressão física entre homens $(1,8 \%)$ foi superior ao observado entre mulheres $(1,3 \%)$.

A maior percepção da exposição à violência pode ser produto da experiência vivida de forma direta, experiência vivida de fato pelo indivíduo, ou de forma indireta, quanto maior a identificação com o grupo estigmatizado como mais vulnerável à violência (Cardia, 1999; Cara \& Gauto, 2007). É importante destacar que ao evidenciar essa representação social da mulher como exposta ao risco não se desconsidera que há reais violências de gênero que afetam diretamente e indiretamente a percepção da violência contra a mulher (Okabe \& Fonseca, 2009). Dados da Fundação Perseu Abramo (2006) demonstram que 33\% das mulheres admitem ter sofrido algum tipo de violência física, $27 \%$ sofreram violências psíquicas e $11 \%$ disseram já ter sofrido assédio sexual. Portanto, destacase que há diferenças em função do sexo quanto à percepção da exposição à violência e do risco e também que são as mulheres quem demonstram a maior percepção do risco (Lordelo, Campos, Fiaes, Ripardo, Cunha, \& Barbosa, 2011).

Os resultados confirmaram parcialmente a hipótese de que a imprevisibilidade familiar na infância relacio- 
na-se com altas taxas de propensão ao risco e baixas taxas de expectativa de futuro. A imprevisibilidade familiar na infância não diferenciou a exposição à violência. $\mathrm{O}$ envolvimento em violência pode funcionar tanto como uma forma de garantir domínio sobre recursos quanto ser um indicador do grau de escassez ambiental do recurso e, portanto, necessidade de competição pela garantia do mesmo. Por exemplo, um estresse vivenciado no ambiente imediato pode ativar a estratégia de orientação em curto prazo e levar indivíduos a assumirem escolhas arriscadas, às vezes aumentando o nível de agressividade e possivelmente execução de comportamentos violentos na tentativa de adquirir status, controlarem os recursos do ambiente, o número de parcerias sexuais (Davis \& Werre, 2008). Considerando a relação do risco com as características do ambiente atual, apontada por Howat-Rodrigues (2013), podemos supor que se sentir exposto à violência ou praticá-la parece ser uma resposta mais adaptada às circunstâncias de imprevisibilidade do ambiente presente do que à imprevisibilidade familiar na infância, pois seguindo as variações sofridas pelo contexto imediato o sujeito pode obter maiores vantagens de sobrevivência. Essa explicação se torna ainda mais favorável ao se observar a amostra, pois supomos que construir uma carreira profissional, encontrar uma namorada/o e ganhar dinheiro são cobranças sociais que compõem a dinâmica do ambiente universitário. Uma segunda explicação se refere à inadequação da prática da violência quanto ao contexto presente, pois exercer um comportamento no momento inadequado às relações sociais pode ser um motivo para que o grupo onde o sujeito agressivo se relaciona o rejeite e, uma vez excluído das relações sociais, o sujeito se torne vulnerável.

A relação entre imprevisibilidade familiar na infância e a expectativa de futuro não se deu como esperado, ao contrário, o grupo com maior imprevisibilidade obteve significativamente as maiores taxas de expectativa de vida. Este resultado, assim como o anterior, também pode estar relacionado à imprevisibilidade atual do ambiente. De acordo com Wilson e Daly (1997) além da influência do modelo mental formado durante a infância, também há a possibilidade da influência da imprevisibilidade objetiva (e.g. violência no bairro de moradia, idade de morte de parentes, etc.) do ambiente imediato do indivíduo sobre a expectativa de vida que este apresenta. De modo geral, os resultados referentes à imprevisibili- dade podem estar relacionados com o fato de que o ambiente se modifica ao longo do tempo trazendo novas perspectivas e expectativas para os indivíduos de forma que seu comportamento não é orientado unicamente pelo modelo de imprevisibilidade que a pessoa vivenciou durante a infância (Daly \& Wilson, 2001).

Houve diferenças entre os grupos de alta imprevisibilidade e baixa imprevisibilidade quanto a um dos indicadores de propensão ao risco. O grupo com maiores taxas de imprevisibilidade familiar na infância foi o que apresentou, significativamente, maiores taxas de risco em competição. Porém era esperado que a alta imprevisibilidade familiar na infância também afetasse o índice de risco em coesão, o que não aconteceu. Esse resultado pode estar relacionado com o fato de que enfrentar desafios e competições para obter algum recurso pode ser entendido como vantajoso para a sobrevivência num ambiente imprevisível. Porém, se ao encontrar o recurso o indivíduo tem que dividi-lo com o grupo (uma das desvantagens da vida em grupo - Lordelo et al., 2011), isso pode significar desvantagem para sobrevivência daquele que competiu. No geral apontamos que a amostra selecionada neste estudo - universitários - que, mesmo apresentando diferenças na imprevisibilidade caracterizam-se pela baixa imprevisibilidade, pode ter contribuído para a ausência de algumas relações esperadas tanto entre homens e mulheres, quanto entre a imprevisibilidade e as outras variáveis estudadas. Sugere-se que estudos futuros trabalhem com amostras heterogêneas quanto à imprevisibilidade familiar na infância a fim de esclarecer as relações entre imprevisibilidade, risco e expectativas de futuro.

Conclui-se que variáveis contextuais e pessoais em conjunto são importantes para o estudo da propensão ao risco, expectativa de futuro e exposição à violência, mesmo apresentando naturezas diferentes. Enquanto a imprevisibilidade está associada a características ambientais que podem mudar ao longo da vida do organismo, diferenças em função do sexo tendem a ser características mais estanques e independentes de mudanças ambientais. Os construtos aqui tratados (propensão ao risco, exposição à violência e expectativa de futuro), entretanto, parecem receber múltiplas influências de outras variáveis não contempladas aqui, tal como a imprevisibilidade do ambiente atual, importante de ser considerada em estudos futuros. Aponta-se também a necessidade de validação do instru- 
mento utilizado na mensuração do desconto do futuro para a população brasileira, a fim de contribuir para a padronização da produção cientifica na área.

\section{REFERÊNCIAS}

Associação Brasileira de Empresas de Pesquisa. (2008). Critério de Classificação de Renda Brasil. Retirado em 16 de abril de 2015 de http://www.abep.org/novo/File Generate. $\operatorname{ash} x$ ?id $=250$

Aldrighi, T. (2004). Prevalência e cronicidade da violência física no namoro entre jovens universitários do Estado de São Paulo - Brasil. Psicologia: teoria e prática, 6(1), 105-120.

Baker, M. D. \& Maner, J. K. (2008). Risk-taking as a situationally sensitive male mating strategy. Evolution and Human Behavior, 29, 391-395.

Baus, J., Kupek, E., \& Pires, M. (2002). Prevalência e fatores de risco relacionados ao uso de drogas entre escolares. Revista Saúde Pública, 36(1), 40-46.

Brandão, E. R. \& Heilborn, M. L. (2006). Sexualidade e gravidez na adolescência entre jovens de camadas médias do Rio de Janeiro, Brasil. Cad. Saúde pública, 22(7), 1421-1430.

Byrnes, J. P., Miller, D. C., \& Schafer, W. D. (1999). Gender differences in risk taking: A meta analysis. Psychological Bulletin. 125(3), 367-383.

Cara, D. \& Gauto, M. (2007). Juventude: percepção de exposição à violência. In Abramovay, M., Andrade, E. R. \& Esteves, L. C. G. (Eds). Juventudes: outros olhares sobre a diversidade (Vol. 27, pp. 172-196). Brasília: Edições MEC/Unesco.

Cardia, N. (1999). Os impactos da exposição à violência: aceitação da violência ou horror continuado? O caso de São Paulo. Trabalho apresentado no Culture, Citizenship and Urban Violence Seminary, Cuernavaca. Resumo retirado de http://www.nevusp.org/portugues/index.php? option=com_content\&task=view\&id=884\&Itemid $=96$

Daly, M., \& Wilson, M. (2001). Risk-taking, intrasexual competition, and homicide. Nebraska Symposium on Motivation, 47, 1-36.

Daly, M., \& Wilson, M. (2005). Carpe diem: Adaptation and devaluing the future. The Quarterly Review of Biology, 80, 55-60.

Dancey, C. P., \& Reidy, J. (2006). Estatística sem matemática para psicologia usando SPSS para Windows. Porto Alegre: Artes Médicas.

Davis, J., \& Were, D. (2008). A longitudinal Study of the effects of uncertainty on reproductive behaviors. Human Nature, 19, 426-452.
Falcão Júnior, J. S. P., Lopes, E. M., Freitas, L. V. de, Rabelo, S. T. de O., Pinheiro, A. B., \& Ximenes, L. B. (2007). Perfil e práticas sexuais de universitários da área de saúde. Escola Anna Nery, 11(1), 58-65. doi: 10.1590/S1414-81452007000100008.

Feijó, R. B., \& Oliveira, E. A. (2001). Comportamento de risco na adolescência. Jornal de pediatria, 77(2), 125134.

Ferreira, J. H. B. P. (2009). Sócio-sexualidade e desconto do futuro: mecanismo de alocação de investimentos $e$ tomada de decisão. (Dissertação de Mestrado). Universidade de São Paulo (USP), São Paulo.

Fetchenhauer, D., \& Rohde, P. A. (2002). Evolutionary personality psychology and victimology: Sex differences in risk attitudes and short-term orientation and their relation to sex differences in victimizations. Evolution and Human Behavior, 23, 233-244.

Fundação Perseu Abramo. (2006). Pesquisa de opinião pública: tipos de violência [em linha]. Fundação Perceu Abramo. Retirado em 10 de julho de 2012, de http:// www.fpabramo.org.br/o-que-fazemos/pesquisas-deopiniao-publica/pesquisas-realizadas/tipo-de-violenciasofrida

Gava, A. M. \& Vieira, K. M. (2008). Risco e gênero: medindo a tolerância ao risco e as diferenças entre os gêneros. Revista de Ciência da Administração, 10(20), 116140.

Hair, J. F. Jr., Anderson, R. E., Thatam, R. L., \& Black, W. C. (2006). Análise multivariada de dados. (5 $5^{\mathrm{a}}$ ed.). Porto Alegre: Bookman.

Hill, E. M., Jenkins, J., \& Farmer, L. (2008). Family unpredictability, future discounting, and risk taking. Journal of Socio-Economics, 37(4), 1381-1396. doi: 10.1016/j.socec.2006.12.081

Howat-Rodrigues, A. B. C. (2010). Percepção de Imprevisibilidade Familiar e sua relação com a Propensão ao Risco e o Desconto do Futuro. (Dissertação de Mestrado). Universidade Federal do Espírito Santo (UFES), Vitória.

Howat-Rodrigues, A. B. C. (2013). Propensão ao risco diante de contextos de imprevisibilidade: uma análise a partir da abordagem evolucionista ao comportamento humano. (Tese de doutorado). Universidade de São Paulo (USP), São Paulo.

Howat-Rodrigues, A. B. C., De Andrade, A. L., \& Tokumaru, R. S. (2012). Construção e validação da Escala de Imprevisibilidade Familiar na Infância (EIFI). Psicologia: Reflexão e Crítica. 25(2), 203-211.

Howat-Rodrigues, A. B. C., De Andrade, A. L., \& Tokumaru, R. S. (2013). Escala de Propensão ao Risco Específico - Domínios Evolutivos (EPRE-DE): adaptação e estrutura fatorial. Avaliação Psicológica. 12(3), 281-290. 
Instituto Brasileiro de Geografia e Estatística. (1999). População jovem no Brasil: A dimensão demográfica [em linha]. Instituto Brasileiro de Geografia e Estatística (IBGE). Obtido em 10 de julho de 2012, de http://www. ibge.gov.br/home/estatistica/populacao/populacao_ jovem_brasil/comentario1.pdf

Instituto Jones dos Santos Neves. (2008). Violência contra a mulher 2008: Ocorrências registradas na delegacia especializada da mulher [DEAM], Vitória - ES 2004 a 2006 [em linha]. Instituto Jones dos Santos Neves. Obtido em 10 de julho de 2012, de http://www.ijsn.es.gov.br/ Sitio/attachments/222_ViolenciacontraMulher.pdf

Kirby, K. N. \& Santiesteban, M. (2003). Concave utility, transaction costs, and risk in measuring discounting of delayed rewards. Journal of Experimental Psychology, 29(1), 66-79.

Koller, S. H. (1999). Resiliência e vulnerabilidade em crianças que trabalham e vivem na rua. Educar em Revista. $15,67-71$.

Krebs, J. R. \& Davies, N. B. (1996). Conflito sexual e seleção sexual. In Krebs, J. R. \& Davies, N. B. (Eds), Introdução à Ecologia Comportamental (pp. 175-207). São Paulo: Atheneu.

Kronbauer, J. F. D. \& Meneghel, S. N. (2005). Perfil da violência de gênero perpetrada por companheiro. Revista Saúde Pública, 39(5), 695-701.

Lima, C. A., Howat-Rodrigues, A. B. C., \& Tokumaru, R. S. (no prelo). Escala de percepção de exposição à violência (EPEV). In Natividade, J. C. (Ed.), Instrumentos em Psicologia Evolucionista.

Lordelo, E. da R., Campos, A. F., Fiaes, C. S., Ripardo, C. R., Cunha, S. A., \& Barbosa, S. de C. O. (2011). Percepção, atratividade e probabilidade de engajamento em situações de risco: diferenças de gênero. Interação Psicologia, 15(1), 11-19.

Lyra, S. M. K., Goldberg, T., \& Iyda, M. (1996). Mortalidade de adolescentes em área urbana da região sudeste do Brasil. Saúde Pública, 30(6), 587-591.

Magar, E. C. E., Phillips, L. H., \& Hosie, J. A. (2008). Self regulation and risk-taking: Personality and individual differences. Elsevier, 45(2), 153-159.

Okabe, I. \& Da Fonseca, R. M. G. S. (2009). Violência contra a mulher: contribuições e limitações do sistema de informação. Estudo Teórico, 43(2), 453-458.

Ornelas, C. O. (2010). Uma análise da amizade sob a perspectiva evolucionista: Influência dos perfis cognitivos $e$ das características pessoais na preferência por potenciais amigos. (Dissertação de Mestrado). Universidade de São Paulo (USP). São Paulo.

Pillon, S. C., O’Brien, B., Chavez, K. A. P. (2005) A relação entre o uso de drogas e comportamentos de risco entre universitários brasileiros. [Número especial] Rev La- tino-am Enfermagem nov./dez. 13:1-8. Obtido de http://www.scielo.br/pdf/rlae/v13nspe2/pt_v13nspe2a11. pdf

Rolim, M. (2009). Pesquisa de vitimização na cidade de Canoas/RS (Relatório de Pesquisa/2009), Canoas, Porto Alegre. Secretaria de Segurança Pública e Cidadania, Prefeitura de Canoas. Marcos Rolim Direitos Humanos e Segurança Pública Consultoria, Instituto Methodos.

Ross, L. T., Hill, E. M., \& Low, B. S. (1997). The role of future unpredictability in human risk-taking. Human nature, 8(4), 287-325.

Ross, L. T., Hill, E. M. (2002). Childhood unpredictability, schemas for future unpredictability, and risk taking. Social Behavior and Personality, 30, 453-474.

Sanchez, Z. V. M., De Oliveira, L. G., \& Nappo, S. A. (2005). Razões para o não-uso de drogas ilícitas entre jovens em situação de risco. Revista Saúde Pública, 39(4), 599-605.

Saraiva, A. M., Quixadá, L. M. (2010) Realização, sofrimento, saúde e adoecimento: algumas reflexões sobre o estudante e sua trajetória universitária. In: Conferência Internacional sobre os Setes Saberes para uma Educação do Presente (Ed.). Anais eletrônicos da Conferência Internacional sobre os Sete Saberes (p. 7). Fortaleza: UECE.

Souza, E. R. (2005). Masculinidade e violência no Brasil: contribuições para reflexão no campo da saúde. Ciência \& Saúde Coletiva, 10(1), 59-70.

Souza, E. R., De Assis, S. G., \& De Silva, C. M. F. P. (1997). Violência no Município do Rio de Janeiro: Áreas de risco e tendências da mortalidade entre adolescentes de 10 a 19 anos. Revista Panamericana de Salud Pública, 1(5), 389-399.

Steinberg, L. (2004). Risk taking in adolescence: What changes, and why? Annals of the New York Academy of Sciences, 1021(1), 51-58.

Surbey, M. K. (1990). Family composition, stress, and the timing of human menarche. In Zeigler, T. E. \& Bercovitch, F. B. (Eds.), The Socioendocrinology of Primate Reproduction (pp. 11-32). New York: WileyLiss.

Trivers, R. L. (1972). Parental investment and sexual selection. In B. Campbell (Eds.), Sexual selection and the descent of man (pp. 1871-1971). Chicago: Aldine.

Vermelho, L. L., Barbosa, R. H. S., \& Nogueira, S. A. (1999). Mulheres com Aids: desvendando histórias de risco. Caderno de Saúde Pública, 15(2), 369-379.

Waiselfisz, J. J. Mapa da violência 2012: os novos padrões da violência homicida no Brasil. Obtido em 22 de setembro de 2013, de http://www.mapadaviolencia.org.br/ mapa2012.php 
Waiselfisz, J. J. Mapa da violência 2013: Homicídios e Juventude no Brasil. Obtido em 22 de setembro de 2013, de http://www.mapadaviolencia.org.br/mapa2013_jovens. php

Wang, X. T., Kruger, D. J., \& Wilke. (2009). A. Life history variables and risk-taking propensity. Evolution and Human Behavior, 30, 77-84.

Wilson, M. \& Daly, M. (1997). Life expectancy, economic inequality, homicide, and reproductive timing in Chicago neighbourhoods. British Medical Journal, 314, 12711274.

Wilson, M, \& Daly, M. (2004). Do pretty women inspire men to discount the future? The Royal Society, 271, 177179.

Wilson, M. \& Daly, M. (2006). Are juvenile offenders extreme future discounters? Psychological Science. 17, 989-994.
Wilson, M., Daly, M., \& Pound, N. (2002). An evolutionary psychological perspective on the modulation of competitive confrontation and risk-taking. In Pfaff, D. W., Arnold, A. P., Etgen, A. M., Rubin R. T. \& Fahrbach, S. E. (Eds.), Hormones, Brain and Behavior (Vol. 5, pp. 381-408). San Diego: Academic Press.

Yunes, J. \& Primo, E. (1983). Características da mortalidade em adolescentes brasileiros das capitais das unidades federadas. Revista Saúde Pública, 17, 263-278.

Recebido em: 22/01/2014 Última alteração em: 20/04/2015 Aceito em: 25/05/2015 